\title{
The role of the striatum in sentence processing: Evidence from a priming study in early stages of Huntington's disease
}

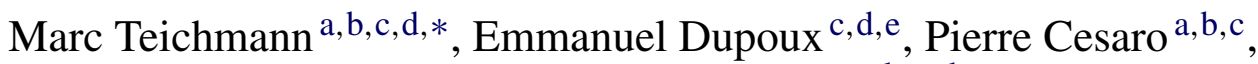 \\ Anne-Catherine Bachoud-Lévi a,b,c,d \\ a INSERM U841, Equipe 1, Neuropsychologie Interventionnelle, Créteil, France \\ ${ }^{\mathrm{b}}$ Université Paris XII, Créteil, France \\ ${ }^{c}$ AP-HP, Hôpital Henri Mondor, Créteil, France \\ ${ }^{\mathrm{d}}$ Ecole Normale Supérieure, Département d'Etudes Cognitives, Paris, France \\ e Laboratoire de Sciences Cognitives et Psycholinguistique, UMR8554, EHESS-ENS-CNRS, Paris, France \\ Received 5 May 2007; received in revised form 24 July 2007; accepted 31 July 2007 \\ Available online 3 August 2007
}

\begin{abstract}
The role of sub-cortical structures such as the striatum in language remains a controversial issue. Based on linguistic claims that language processing implies both recovery of lexical information and application of combinatorial rules it has been shown that striatal damaged patients have difficulties applying conjugation rules while lexical recovery of irregular forms is broadly spared (e.g., Ullman, M. T., Corkin, S., Coppola, M., Hickok, G., Growdon, J. H., Koroshetz, W. J., et al. (1997). A neural dissociation within language: Evidence that the mental dictionary is part of declarative memory, and that grammatical rules are processed by the procedural system. Journal of Cognitive Neuroscience, 9(2), 266-276). Here we bolstered the striatum-rule hypothesis by investigating lexical abilities and rule application at the phrasal level.

Both processing aspects were assessed in a model of striatal dysfunction, namely Huntington's disease (HD). Using a semantic priming task we compared idiomatic prime sentences involving lexical access to whole phrases (e.g., "Paul has kicked the bucket") with idiom-derived sentences that contained passivation changes involving syntactic movement rules (e.g., "Paul was kicked by the bucket"), word changes (e.g., "Paul has crushed the bucket") or either. Target words that were either idiom-related (e.g., "death") reflecting lexical access to idiom meanings, word-related (e.g., "bail") reflecting lexical access to single words, or unrelated (e.g., "table").

HD patients displayed selective abnormalities with passivated sentences whereas priming was normal with idioms and sentences containing only word changes. We argue that the role of the striatum in sentence processing specifically pertains to the application of syntactic movement rules whereas it is not involved in canonical rules required for active structures or in lexical processing aspects. Our findings support the striatum-rule hypothesis but suggest that it should be refined by tracking the particular kind of language rules depending on striatal computations.
\end{abstract}

(C) 2007 Elsevier Ltd. All rights reserved.

Keywords: Striatum; Syntax; Rules; Huntington's disease

\section{Introduction}

While the linguistic role of cortical areas is relatively well established, the role of sub-cortical structures such as the striatum and the way they impact on language processing is still controversial. Indeed, studies investigating striatal damaged patients have shown various kinds of language disorders

\footnotetext{
* Corresponding author at: AP-HP, Hôpital Henri Mondor, Créteil, France. Tel.: +33 6 66263919/1 49812309.

E-mail address: marcteichmann@ hotmail.com (M. Teichmann).
}

ranging from semantic disorganisation to syntactic impairment in both neurodegenerative diseases such as Huntington's and Parkinson's disease (e.g., Copland, 2003; Frank, McDade, \& Scott, 1996; Illes, 1989) and in patients with vascular damage (e.g., Damasio, Damasio, Rizzo, Varney, \& Gersh, 1982; Wallesch \& Papagno, 1988). This might be related to the different lesion patterns regarding the different aetiologies: vascular disorders affect various parts of the striatum and often involve surrounding white matter fibre tracks, Parkinson's disease (PD) is due to neural degeneration in the substantia nigra resulting in dopamine-related dysfunction of the striatum, whereas Huntington's disease (HD) is characterised by neuronal death that 
specifically originates in the neostriatum comprising the caudate head and the putamen (see Peschanski, Cesaro, \& Hantraye, 1995 for a review). Thus, only early stages of HD might represent a reliable model of near exclusive striatal disorders (Kuhl et al., 1982; Mazziotta et al., 1987). Yet, investigations of healthy adults, by means of functional brain imaging, have not clarified the picture either. They showed striatal activation with tasks that were drawn from various language domains including phonology (Tettamanti et al., 2005), word morphology (Vannest, Polk, $\&$ Lewis, 2005) and syntax (e.g., Friederici \& Kotz, 2003; Moro et al., 2001). Indeed, this functionally unspecified picture has led to two competing views. On the one hand, it has been argued that the striatum merely underpins general non-language specific processes such as executive functioning, which is thought to supervise and modulate linguistic information (e.g., Grossman et al., 2000, 2002; Longworth, Keenan, Barker, Marslen-Wilson, \& Tyler, 2005). Conversely, several authors have proposed that the striatum subserves particular kinds of language computations that may cut across different linguistic levels, including phonology, morphology and syntax (e.g., Ullman et al., 1997; Ullman, 2001). According to this latter view, the striatum was claimed to impact on the computation of combinatorial language rules that apply to various kinds of lexically stored information. This proposal is derived from psycholinguistic models that divide the human language faculty into two core components comprising a mental lexicon and a computational grammar. The lexicon contains all linguistic idiosyncrasies such as phonemes, morphemes, words and even whole phrases (e.g., idioms), whereas the computational grammar holds the combinatorial rules that are applied to the lexical input (Chomsky, 1965; Pinker, 1999).

The Ullman proposal, according to which the striatum is specifically involved in rule computations has been mainly assessed in the domain of word morphology by comparing the conjugation of regular verbs or non-verbs (rule-based; e.g., walk/walk-ed or splush/splush-ed) and of irregular verbs (lexical-based; e.g., go/went). However, studies using striatal damaged patients such as PD, HD and stroke patients yielded conflicting views. Ullman et al. (1997) showed that these patients are mainly impaired on the conjugation of regular verbs and non-verbs whereas their performance on irregular verbs is near-normal. They concluded that the striatum subserves the application of the English past tense rule, which was thought to substantiate the striatum-rule theory. Teichmann et al. (2005), using the richer morphology of French with HD, restricted this view to conjugation sub-rules, while Longworth et al. (2005) using a priming study with regular and irregular prime-target pairs fully rejected the striatum-rule theory. Such conflicting data might be due to the different rules assessed in different languages, to the different tasks and/or to the differences of the respective lesion patterns. Thus, to further clarify this issue, the general claim of the striatum-rule hypothesis should be tested in a second language domain extending evidence from the word level to the level of sentence processing.

In this vein, several studies have investigated the link between the striatum and syntactic processing aspects. However, they did not compare syntactic rules and lexical operations in a direct way. With respect to rule application, most authors used noncanonical sentences like passives (e.g., "the boy was kissed by the girl") or object-relatives (e.g., "the boy that the girl kissed"), in which the usual agent-action-theme order (subjectverb-complement) is inverted as compared to actives ("the boy kissed the girl") or subject-relatives ("the boy that kissed the girl"). According to several linguistic accounts (e.g., Chomsky, $1965,1977,1986)$ such non-canonical sentences involve the application of syntactic movement rules that allow mapping of the non-canonical surface structure (e.g., "the boy[3: theme] was

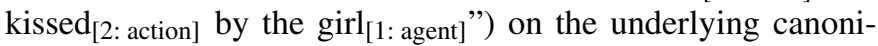
cal structure ("the girl[1: agent] kissed [2: action] the boy[3: theme]"). These rules are thought to be critical for assigning thematic roles to the different words and thus to convey the correct sentence meaning ("who [agent] does what [action] to whom [theme]"). Note that even if the initial concept of grammatical movement has been revised in more recent theories, the notion of grammatical transformation always holds a central role in almost all models of generative grammar. When studying this canonical versus non-canonical contrast several studies have shown that PD patients have difficulties interpreting non-canonical word order whereas they have near normal performance on canonical structures, such as actives and subject-relatives (Kemmerer, 1999; McNamara, Krueger, O’Quin, Clark, \& Durso, 1996; Natsopoulos et al., 1993). Similar results were reported in early stages of HD. Teichmann et al. (2005) showed that these patients were massively impaired on sentence comprehension when the application of syntactic movement rules was required for accurate response. The authors independently varied the plausibility and the canonicity of simple sentences (e.g., canonical plausible: "the girl waters the flower", non-canonical non-plausible: "the girl is watered by the flower"), which were presented with a picture that depicted either the corresponding scene or the inverted one (e.g., "a girl that waters a flower" or "a flower that waters a girl"). HD patients had severe difficulties in choosing the correct picture when confronted with non-canonical sentences especially when non-plausible sentence meanings impeded the compensatory use of pragmatic reasoning.

In contrast to these previous studies exclusively assessing syntactic rule application, several authors evaluated striatal involvement in lexical aspects of sentence processing. Most of them assessed the processing of grammatical categories in phrasal contexts using functional brain imagery with healthy adults. Indeed, grammatical categories are claimed to be stored in the mental lexicon together with the respective word representation (e.g., cat-noun, eat-verb, the -determiner, ... (MacDonald, Pearlmutter, \& Seidenberg, 1994). Furthermore, certain grammatical categories such as determiners place constraints on the following sentence word (e.g., "determiners" are always followed by "nouns"), which defines a specific grammatical expectancy. The kind of grammatical expectancy was used in a PET study of Moro et al. (2001) who inverted determiners and nouns in sentences that consisted of pseudowords so as to neutralise the access to semantic components while maintaining function words. They reported activation of the caudate head when participants covertly read such sentences and subsequently made acceptability judgements. Likewise, in 
a functional MRI study Friederici, Ruschemeyer, Hahne, \& Fiebach (2003) showed that violations of the expectancy of grammatical categories (e.g., "the ice cream was in the *eaten") results in the activation of the left putamen. Similar sentence materials were also used with striatal damaged patients during ERP recollection (PD, vascular disorders). Several authors showed that the P600 component, which was hypothesised to index late stages of grammatical integration, is either absent (Friederici, Kotz, Werheid, Hein, \& von Cramon, 2003; Kotz, Frisch, von Cramon, \& Friederici, 2003) or reduced in these patients (Friederici, von Cramon, \& Kotz, 1999). Such findings led to the assumption that the striatum subserves controlled post-linguistic processes allowing to verify and to integrate the output of early and automatic stages of syntactic processing (Friederici \& Kotz, 2003). This proposal was further specified by Longworth et al. (2005) suggesting that the striatum inhibits competing alternatives at late stages of grammatical integration and, possibly, in the the lexicalization stage of language processing (Longworth et al., 2005). Indeed, impaired inhibition due to striatal damage might account for the incorrect integration of lexically stored information such as grammatical categories, which, in turn, might reduce the $\mathrm{P} 600$ in striatal damaged patients. However, intriguingly, this lexical and post-linguistic view of the striatum seems to be restricted to the processing of grammatical categories. Indeed, the investigation of other domains that heavily depend on lexicalised information, such as semantics, showed that striatal damaged patients display a normal N400 component, a marker of semantic incongruence, when tested with sentences that contained semantic restriction violations (e.g., "the volcano was eaten”) (Friederici et al., 1999; Friederici, Kotz et al., 2003; Kotz et al., 2003).

In summary, the previous studies suggest that the striatum plays a role in sentence processing but it has remained controversial as to whether this role is tied to the rule aspect, to lexical operations or to both. Furthermore, as rules and lexical processes were not contrasted in a direct way, it has remained unclear whether the striatum genuinely impacts on linguistic aspects of sentence processing or whether it operates via nonlanguage specific processes that may concomitantly affect both rules and the lexicon.

The current study addressed these issues in a model of striatal disorders, namely early stages of HD, using a task that directly compared lexical and rule aspects through the contrast of idiomatic and non-canonical sentences. According to several linguistic accounts, idiomatic sentences such as "[Paul] kicked the bucket" are stored in the mental lexicon as extended language chunks (Chomsky, 1980; Swinney \& Cutler, 1979). Indeed, access to the 'arbitrary' idiom meaning ("[Paul] has died") does not depend on syntactic rules, the application of which would convey a literal sentence meaning ("[Paul] has 'crushed' the bucket"). Several studies have tested this issue with healthy adults using a semantic priming paradigm, in which idiomatic prime sentences (e.g., “. . . her goldfish kicked the bucket”) preceded target words that were either related to the figurative idiom meaning (e.g., 'die') or to the meaning of the last sentence word (e.g., 'bail') (Cacciari \& Tabossi, 1988; Titone \& Connine 1994). They found that lexical decisions (word versus non-word) were faster on both word-related and idiom-related target words as compared to unrelated targets (e.g., 'lie'), showing that idiom processing involves lexical access to both the component words and the figurative idiom meaning. In the present study we used a similar priming task manipulating idioms (e.g., "Paul has kicked the bucket") as well as idiom-derived sentences that contained passivation changes involving movement rules (e.g., "Paul was kicked by the bucket"), word changes (e.g., "Paul has crushed the bucket") or either (e.g., "Paul was crushed by the bucket"). This allowed assessing both lexical accesses to idiom and to word meanings as well as the ability to apply syntactic movement rules. HD patients were predicted to have normal performance with idioms and with idiom-derived sentences containing only word changes but not with passivated idiom-derived sentences, which imply the application of movement rules. In particular, we expected them to process these latter sentences in a linear manner. So, sentences like "PAUL (was) KICKED (by) THE BUCKET" were thought to yield an idiomatic sentence interpretation, whereas controls were predicted to disrupt this interpretation through the application of movement rules. Results of HD patients were furthermore correlated with general, non-linguistic, parameters including executive function scores as well as markers of disease progression including motor scores and overall functional capacities.

\section{Methods}

\subsection{Participants}

Twenty-four French speaking, early stage, self-sufficient HD patients, classified as stage I according to the "total functional capacity scale" (Shoulson, 1981), and 24 healthy volunteers participated in this study. HD patients were recruited from the out-clinic patients within the programs of interventional therapy and the search for biomarkers, approved by the ethics committee of the Henri Mondor Hospital. HD patients had no previous neurological or psychiatric history other than HD and neurological diagnosis was genetically confirmed (CAG repeats $>35$ ). Healthy controls had no neurological or psychiatric disorders and were matched to the HD patients according to their age and educational level (all $F \mathrm{~s}<1$ ). All participants gave informed consent. Demographic data are summarised in Table 1.

\subsection{General assessment}

All patients were evaluated using the unified Huntington's disease rating scale (UHDRS; Huntington Study Group, 1996) and the Mattis dementia rating scale (MDRS; Mattis, 1976). The UHDRS comprises the evaluation of functional ability (total functional capacities; TFC), of motor functioning (UHDRS motor part) and of executive parameters such as literal fluency, the Stroop test and the digit symbol code (UHDRS cognitive part). General assessment is summarised in Table 2 .

Table 1

Demographic data of HD patients and controls

\begin{tabular}{lll}
\hline & HD & Controls \\
\hline$N$ & 24 & 24 \\
Sex & $13 \mathrm{M} / 11 \mathrm{~F}$ & $12 \mathrm{M} / 12 \mathrm{~F}$ \\
Age (years) & $44.4 \pm 10.9$ & $41.7 \pm 7.9$ \\
Years of education & $12.7 \pm 3.1$ & $13.9 \pm 2.3$ \\
Handedness & $22 \mathrm{R} / 2 \mathrm{~L}$ & $23 \mathrm{R} / 1 \mathrm{~L}$ \\
Evolution duration (years) & $5.5 \pm 3.4$ & - \\
CAG repeats & $44.4 \pm 3.3$ & - \\
\hline
\end{tabular}


Table 2

Results for the general assessment in HD patients

\begin{tabular}{lcc}
\hline & HD & $\begin{array}{l}\text { Normal published } \\
\text { range }\end{array}$ \\
\hline Total functional capacity (TFC) & $12.0 \pm 0.9$ & 13 \\
UHDRS motor score & $27.0 \pm 16.1$ & 0 \\
MDRS & $131.2 \pm 9.6$ & $>136$ \\
Executive functions & & \\
$\quad$ Stroop colour/words & $28.6 \pm 11.0$ & $>35^{\mathrm{a}}$ \\
$\quad$ Fluency PRV (2 min) & $47.2 \pm 24.0$ & $>56^{\mathrm{b}}$ \\
$\quad$ Symbol digit code & $28.9 \pm 12.8$ & $>37^{\mathrm{c}}$ \\
\hline
\end{tabular}

MDRS: Mattis dementia rating scale; Stroop colour/words: interference part of the Stroop test; fluency PRV: mean number of words P, R, V in $2 \mathrm{~min}$.

a Norms are issued from Golden (1978).

b Norms are issued from Cardebat, Doyon, Puel, Goulet, \& Joanette (1990).

c Norms are issued from Wechsler (1981).

\section{Priming task with idioms and idiom-derived sentences}

We used a semantic priming task, in which auditory prime sentences were followed by visually presented target words. Participants performed lexical decisions on the targets (word versus non-word decision). The main characteristic of semantic priming is that a prime (e.g., "He has kicked the bucket") facilitates the recognition of semantically related targets (e.g., "bail" or "death") as compared to semantically unrelated targets (e.g., "table"). Such priming paradigms have a theoretical advantage over classical sentence comprehension tasks in that they do not depend on explicit comprehension of the sentence materials. Thus, they diminish the impact of controlled processes and allow assessing more implicit language-specific aspects of sentence processing.

Prime sentences included idioms (e.g., "Paul has kicked the bucket") and different idiom-derived sentences, in which the word order was maintained, but either the syntactic structure was changed (passivation), a content word was changed or both were modified. This yielded idioms with passivation (Pidioms; "Paul was kicked by the bucket"), active non-idioms, in which a content word was changed (NI-actives; "Paul has crushed the bucket") and non-idioms with both passivation and content word change (NI-passives; "Paul was crushed by the bucket"). Each prime sentence was paired with an idiomrelated target (e.g., "death"), a word-related target (e.g., "bail"), an unrelated target (e.g., "table") and a non-word target (e.g., "neurre").

We reasoned that idioms convey a figurative meaning, which should result in priming for idiom-related targets. Conversely, such priming effects should be disrupted in idiom-derived sentences as these convey literal sentence meanings either because of syntactic rule application in passivated versions or because of lexical processing in sentences with content word change. Furthermore, we assumed that semantic restriction violations in passivated versions (a 'bucket' can neither 'kick' nor 'crush') should hinder the integration of the verb's arguments (see Schriefers, Friederici, \& Rose, 1998; Tyler, 1985) and thus yield diminished priming for word-related targets. Under the hypoth- esis that HD patients display rule disorders but intact lexical abilities, we predicted them to be insensitive to passivation but sensitive to content word changes. Hence HD patients were predicted to disrupt priming for idiom-related targets only with NI-actives and NI-passives but not with P-idioms. Furthermore, they were believed not to detect semantic restriction violations in rule-dependent passivated versions and thus to display undiminished priming for word-related targets with P-idioms and NI-passives. On the contrary, intact lexical abilities in HD at both the word level and the sentence level were thought to yield normal priming magnitudes for word-related targets with idioms and NI-actives and for idiom-related targets with idioms, respectively.

In order to further explore lexical abilities in HD we verified whether these patients have intact access to semantic restrictions in simple active clauses (e.g., *"Paul has eaten his car" (SR-) versus "Paul has driven his car" (SR+). These clauses were only paired with word-related targets (e.g., "truck") in order to limit the amount of the sentence materials. Like controls, HD patients were predicted to display increased reaction times with sentences that contain semantic restriction violations as compared to sentences that do not.

\subsection{Materials}

We constructed two experimental sets that contained idioms and idiom-derived sentences (main-set) and simple active clauses that did or did not contain semantic restriction violations (sub-set). Both sets are summarised in Table 3.

The main-set was composed of 10 French idioms, from which we derived 10 P-idioms (by passifying the source idiom and inverting the agent and the theme role), 10 NI-actives and 10 NI-passives. Each of the 40 prime sentences were paired with an idiom-related target, a word-related target and an unrelated target as well as with a target non-word. This resulted in a total of 160 prime-target pairs. The four sentence types were matched one with another (all $P$ 's $>0.1$ ) for auditory duration (idioms $1315 \mathrm{~ms}( \pm 114), \mathrm{P}$-idioms $1314 \mathrm{~ms}( \pm 133)$, NI-actives $1393 \mathrm{~ms}( \pm 118)$, NI-passives $1338 \mathrm{~ms}( \pm 79))$ and number of phonemes (idioms: $18.2( \pm 2)$, PI-idioms: $18.2( \pm 2)$, idiomsDA: $18( \pm 2)$, idioms-DP: $18( \pm 2))$. Idioms and NI-actives contained five words while P-idioms and NI-passives contained six words including the French function word "par" (by). Target words were matched one with another (all $P$ 's $>0.1$ ) for their number of letters (idiom-related: $7.6( \pm 3)$, word-related: 6.6 $( \pm 2)$, unrelated: $7.6( \pm 2.4)$, non-word targets: $6.6( \pm 1.2))$ and of phonemes (idiom-related: $6.2( \pm 2.9)$, word-related: $5( \pm 1.9)$, unrelated: $5.9( \pm 2.2)$, non-word targets: $4.7( \pm 0.8))$. Furthermore, idiom-related targets, word-related targets and unrelated target words were matched one with another (all $P$ 's $>0.1$ ) for their log-transformed token frequency according to the LEXIQUE 2 database (New, Pallier, Brysbaert, \& Ferrand, 2004) (idiom-related targets 50 per million $( \pm 107.2)$, word-related targets 28.3 per million $( \pm 38.6)$, unrelated targets 36 per million $( \pm 89.2))$.

The sub-set contained 30 active clauses. Half of them contained semantic restriction violations $(\mathrm{SR}-)$ while the other half 
Table 3

The different types of prime sentences and their respective targets

\begin{tabular}{|c|c|c|c|c|c|c|c|}
\hline \multirow[t]{2}{*}{ Set } & \multirow[t]{2}{*}{ Prime types } & \multirow[t]{2}{*}{ Examples } & \multirow[t]{2}{*}{$N$} & \multicolumn{4}{|l|}{ Target types } \\
\hline & & & & Word-related & Idiom-related & Unrelated & Non-word \\
\hline \multirow[b]{3}{*}{ Main-set } & Idioms & Paul a cassé sa pipe & 10 & \multirow[b]{3}{*}{ Cigare, bail $^{\mathrm{a}}$} & \multirow[b]{3}{*}{ Mort, death } & \multirow[b]{3}{*}{ Table, table } & \multirow[b]{3}{*}{ Neurre, neurr } \\
\hline & P-idioms & Paul est cassé par sa pipe & 10 & & & & \\
\hline & NI-actives & $\begin{array}{l}\text { Paul was kicked by the bucket } \\
\text { Paul a brisé sa pipe }\end{array}$ & 10 & & & & \\
\hline \multirow[b]{2}{*}{ Sub-set } & $\mathrm{SR}+$ & Paul a conduit sa voiture & 15 & \multirow[b]{2}{*}{ Camion, truck } & \multirow[b]{2}{*}{-} & \multirow[b]{2}{*}{-} & \multirow[b]{2}{*}{ Neval, neval } \\
\hline & SR- & $\begin{array}{l}\text { Paul has driven his car } \\
\text { Paul a mangé sa voiture } \\
\text { Paul has eaten his car }\end{array}$ & 15 & & & & \\
\hline
\end{tabular}

$\mathrm{SR}+$ : no semantic restriction violation; $\mathrm{SR}-$ : semantic restriction violation.

a The French word cigare is semantically related to pipe just as bail is related to bucket.

did not (SR+). Each sentence was paired with a word-related target and with a target non-word. This resulted in a total of 60 sentence-target pairs. Both sentence types were matched $(P>0.1)$ for their auditory duration $(\mathrm{SR}+: 1354 \mathrm{~ms}( \pm 81), \mathrm{SR}-$ : $1396( \pm 99))$, number of words (SR+: $5( \pm 0), \mathrm{SR}-: 5( \pm 0))$ and number of phonemes (SR+: $18.6( \pm 3)$, SR-: $19.4( \pm 3.4))$. Furthermore, word targets and non-word targets were matched $(P>0.1)$ for their number of letters (respectively $6.3( \pm 0)$ and $6.2( \pm 0)$ ) and phonemes (respectively $5.3( \pm 3.4)$ and 5.5 $( \pm 2.8)$ ).

Finally, we added 20 passive filler prime sentences in order to obtain an equivalent number of passive and active sentences within the stimuli materials. Ten filler sentences were paired with unrelated targets and 10 were paired with target non-words. All non-word stimuli of the experiment were constructed by changing the first phoneme of existing French nouns, which were distinct from the target words, and consisted of legal phoneme and letter strings.

The four sentence types of the main-set and their respective targets were separated into four blocks (idioms, P-idioms, NI-actives, NI-passives). Fifteen stimuli of the sub-set and 5 passive filler stimuli were added to each of the 4 blocks yielding a total of 60 stimuli per block. The idiom and the Pidiom block contained eight SR+ and seven SR- stimuli of the Sub-set while the NI-actives and the NI-passives blocks contained each seven SR+ and eight SR- stimuli. Overall, within each block $66 \%$ of the stimuli belonged to the same sentence type (respectively idioms, P-idioms, NI-actives and NIpassives) while the other $33 \%$ of the stimuli were not derived from idioms (fillers, active clauses). Furthermore, within each block $69 \%$ of the targets were semantically related to either the last sentence's word or the idiom meaning, whereas there was no semantic link for the remaining $31 \%$ of the targets. We chose a blocked presentation because a pilot study had revealed that mixing idiom and idiom-derived sentences led to high expectancy of idiomatic meaning, which was reflected by undifferentiated priming effects for idiom-related targets with all sentence types.

\subsection{Procedure and apparatus}

Each stimulus trial consisted of the presentation of a fixation cross (+) in the middle of the computer screen for $1000 \mathrm{~ms}$ followed by an auditory prime sentence that was displayed over headphones. The visual target word was presented $200 \mathrm{~ms}$ after the offset of the prime sentence on the computer screen (black uppercase letters, white background) and was centred in the same position as the fixation cross. Participants were instructed to decide as accurately and as quickly as possible whether the target letter string was a real French word or not. They were told to press the button corresponding to their dominant hand if the target was a real word and the button corresponding to their nondominant hand if it was not. Reaction times were measured from the target onset. Targets remained on the computer screen until the participant responded. A new trial was initiated $1000 \mathrm{~ms}$ after response. The stimuli were presented in four successive blocks (idioms, P-idioms, NI-actives, NI-passives) the presentation order of which was permuted across the 24 controls and across the $24 \mathrm{HD}$ patients. Within the blocks stimuli were presented randomly for each participant. Each of the four blocks was followed by a short pause and the subsequent block was initiated when the participant felt ready. All prime sentences were recorded by a native French male speaker and digitised for binaural presentation over headphones using COOL EDIT software. The experiment was administered on an IBM laptop computer using E-PRIME experimental software.

\section{Results}

Analyses of variance (ANOVAs) were conducted by participants $(F 1)$ and by items $(F 2)$ with reaction time (RT) as the dependent variable. They were conducted separately for the two experimental sets. For each participant, incorrect responses and missing data were excluded from the analyses. Furthermore, we calculated mean reaction times and standard deviations for each participant and excluded data that deviated by more than two standard deviations from the mean values for a given partici- 

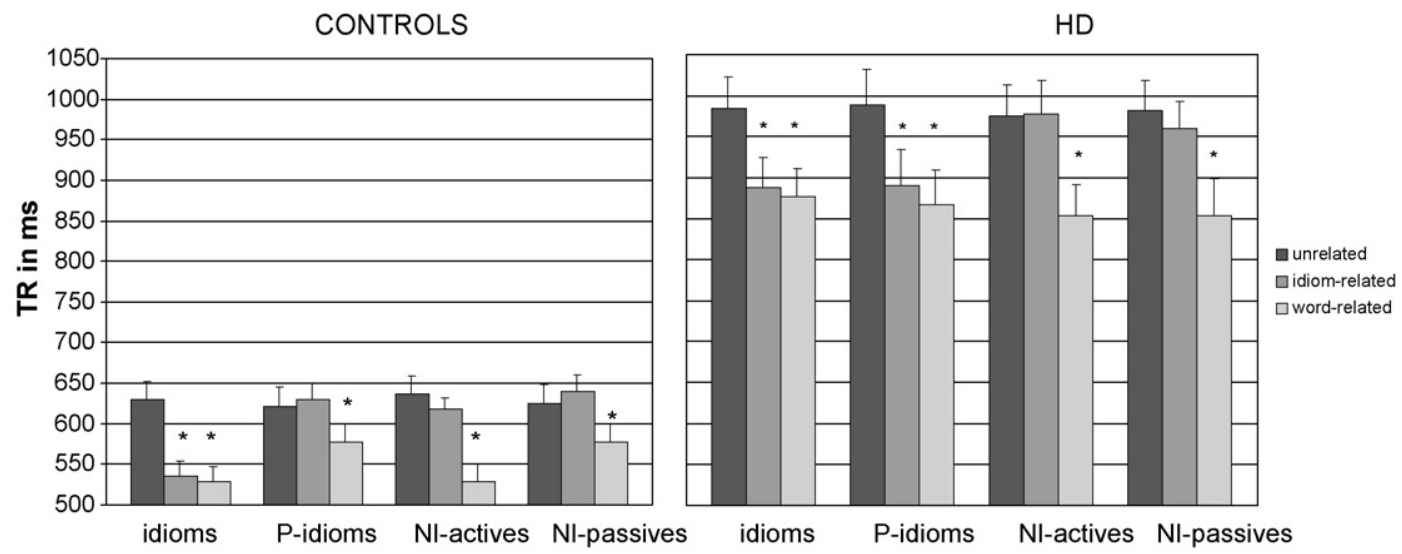

Fig. 1. Experimental main-set: mean reaction times and standard deviations with the different prime and target types in Controls and HD patients. * $P$ is significant at the 0.05 level for the comparison between unrelated and related targets.

pant. This yielded the exclusion of 5.6\% data from the main-set (HD: $7.1 \%$, controls: $4.1 \%$ ) and of $3.5 \%$ data from the sub-set (HD: $5.8 \%$, controls: $1.3 \%$ ).

\subsection{Main-set}

We used the three independent variables "group" (HD, controls), "prime type" (idioms, P-idioms, NI-actives, NI-passives) and "target type" (idiom-related, word-related, unrelated). Fig. 1 summarises the RT with the different prime and target types for controls and HD patients.

RT were slower in HD $(925 \mathrm{~ms} \pm 198)$ than in controls $(596 \mathrm{~ms} \pm 108) \quad(F 1(1,46)=72.06, \quad P<0.001 ; \quad F 2(1$, $108)=3760.88, P<0.001)$. There was a target type effect (word related $708 \mathrm{~ms} \pm 220$, idiom-related $767 \mathrm{~ms} \pm 222$, unrelated $806 \pm 235)(F 1(2,92)=153.34, P<0.001 ; F 2(2$, $108)=44.63, P<0.001$ ) and a prime type effect (idioms $741 \mathrm{~ms} \pm 231$, P-idioms $763 \pm 228$, NI-actives $765 \pm 234$, NIpassives $773 \pm 224)$ in the analysis by items $(F 2(3,108)=3.43$, $P=0.02)$ but not in the analysis by participants ( $F 1(3$, $138)=2.09, P>0.1)$. There was a prime type $\times$ target type interaction $(F 1(6,276)=7.80, P<0.001 ; F 2(6,108)=3.68$, $P=0.002)$ as well as a triple group $\times$ prime type $\times$ target type interaction $(F 1(6,276)=2.85, P=0.010 ; F 2(6,108)=3.24$, $P=0.006)$. Restricted analyses compared RT for idiom-related and word-related targets with RT for unrelated targets (priming effects). Results with idioms, NI-actives and NI-passives were similar in HD patients and controls. With idioms, RT were faster for both idiom-related targets (controls: $F 1(1,23)=99.04$, $P<0.001 ; F 2(1,18)=32.86, P=0.001$. HD: $(F 1(1,23)=47.00$, $P<0.001 ; F 2(1,18)=10.33, P<0.005)$ and word-related targets (controls: $F 1(1,23)=91.16, P<0.001 ; F 2(1,18)=49.45$, $P<0.001$. HD: $(F 1(1,23)=55.30, P<0.001 ; F 2(1,18)=9.54$, $P=0.006)$. With NI-actives, RT were similar for idiom-related targets (controls: $(F 1(1,23)=2.20, P>0.1 ; F 2(1,18)=2.11$, $P>0.1$. HD: both $F$ 's $<1)$ but faster for word-related targets (controls: $F 1(1,23)=93.66, P<0.001 ; F 2(1,18)=36.68$, $P<0.001$. HD: $(F 1(1,23)=30.90, P<0.001 ; F 2(1,18)=24.37$, $P<0.001)$. Likewise, with NI-passives, RT were similar for idiom-related targets (controls: $F 1(1,23)=2.86, P>0.1 ; F 2(1$,
$18)=1.55, P>0.1$. HD: both $F$ 's $<1)$ but faster for wordrelated targets (controls: $F 1(1,23)=27.95, P<0.001 ; F 2(1$, $18)=12.56, P=0.002$. HD: $F 1(1,23)=34.28, P<0.001 ; F 2(1$, $18)=11.96, P=0.003$ ). Results with P-idioms were different in controls and HD patients: RT for idiom-related targets were similar in controls (both $F$ 's $<1$ ) but faster in HD patients $(F 1(1,23)=21.80, P<0.001 ; F 2(1,18)=10.28, P=0.005)$. RT for word-related targets were faster in both controls $(F 1(1$, $23)=26.52, P<0.001 ; F 2(1,18)=6.29, P=0.02)$ and $\operatorname{HD}(F 1(1$, $23)=21.29, P<0.001 ; F 2(1,18)=16.58, P=0.001)$. Taken together, controls displayed priming for idiom-related targets with idioms alone, whereas HD patients displayed priming with idioms and P-idioms. Priming for word-related targets existed with all sentence types in both controls and HD patients.

In order to compare the magnitude of the priming effects we calculated the amount of priming in milliseconds for idiomrelated targets $\left(\mathrm{RT}_{\text {unrelated }}-\mathrm{RT}_{\text {idiom-related }}\right)$ and word-related targets $\left(\mathrm{RT}_{\text {unrelated }}-\mathrm{RT}_{\text {idiom-related }}\right)$ and entered them in a second series of ANOVA's (see Table 4). For idiom-related targets priming with idioms was similar in controls and HD (both $F$ 's $<1$ ). Priming for word-related targets was similar in controls and HD patients with idioms (both $F$ 's $<1$ ) and NI-actives (both $F$ 's $<1)$ but not with P-idioms $(F 1(1,46)=9.57, P=0.003$; $F 2(1,9)=6.36, P=0.033)$ and NI-passives $(F 1(1,46)=7.38$, $P=0.009 ; F 2(1,9)=10.85, P=0.009)$. This was related to the fact that priming in controls was smaller with P-idioms than

Table 4

Amount of priming in milliseconds for idiom-related targets and word-related targets in idioms, P-idioms, NI-actives and NI-passives

\begin{tabular}{llllcc}
\hline & \multicolumn{2}{l}{ Unrelated - idiom-related } & & \multicolumn{2}{c}{ Unrelated - word-related } \\
\cline { 2 - 3 } \cline { 6 - 7 } \cline { 5 - 6 } & Controls & HD & & Controls & HD \\
\hline Idioms & $95^{*}$ & $97^{*}$ & & $102^{*}$ & $108^{*}$ \\
P-idioms & -8 & $\mathbf{9 9}^{*}$ & & $44^{*}$ & $\mathbf{1 2 2}^{*}$ \\
NI-actives & 18 & -3 & & $108^{*}$ & $122^{*}$ \\
NI-passives & -15 & 22 & & $47^{*}$ & $\mathbf{1 2 9}^{*}$ \\
\hline
\end{tabular}

Asterisks indicate significant priming (idiom-related/word-related vs. unrelated). Bold characters indicate priming effects that differentiate HD from controls: undisrupted priming for idiom-related targets in P-idioms and undiminished priming for word-related targets in P-idioms and NI-passives. 


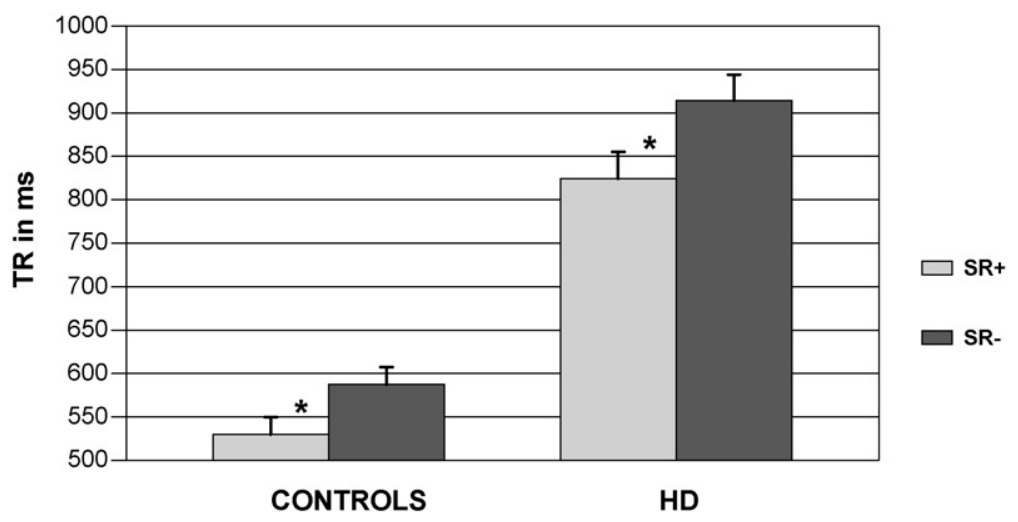

Fig. 2. Sub-set: mean reaction times and standard deviations in controls and HD patients with simple active clause sentences that either respect (SR+) or violate (SR-) semantic restrictions. HD patients are sensitive to semantic restriction violations showing that they have lexical access to verb-specific information in phrasal contexts.

with idioms $(F 1(1,23)=16.67, P<0.001 ; F 2(1,18)=6.91$, $P=0.017)$ and with NI-passives than with NI-actives $(F 1(1$, $23)=20.75, P<0.001 ; F 2(1,18)=6.90, P=0.017)$, whereas there was no difference in HD (all $F$ 's $<1$ ).

Finally, we checked whether the blocked design of the study might have resulted in the use of conscious strategies during sentence processing. First, participants might have changed behaviour within the same block when becoming progressively aware that $66 \%$ of the stimuli belong to the same sentence type. Thus, we ran a post-hoc ANOVA with the variable "intra-block order" comparing the first half of idioms/idiom-derived sentences (first seen) with the second half (second seen). Results indicated that there was no intra-block order effect (both $F$ 's $<1$ ) and that intra-block order did not interact either with "group" (both $F$ 's $<1)$ or with "prime type" $(F 1<1 ; F 2(3,108)=1.58$, $P>0.1)$ or "target type" $(F 1(2,92)=1.97, P>0.1 ; F 2<1)$. There were also no triple or quadruple interactions of the intra-block order with the variables "group", "prime type" and "target type" (all $F$ 's $<1$ ). Second, participants might have displayed different behaviour as a function of the overall block order especially when the idiom block was presented first. We therefore ran post-hoc ANOVA's by subjects for each of the four prime types with the variable "block order" (first seen block, second seen block, third seen block, forth seen block). Analyses showed that there was no block order effect (idioms: $F<1$; P-idioms: $F<1$; NI-actives: $F<1$; NI-passives: $F(3,40)=1.03, P>0.1$ ). Moreover, "block order" neither interact with "group" (idioms: $F<1$; P-idioms: $F(3,40)=1.19, P>0.1$; NI-actives: $F<1$; NIpassives: $F(3,40)=2.16, P>0.1)$ nor with "target type" (idioms: $F<1$; P-idioms: $F(6,80)=2.14, P=0.06$; NI-actives: $F<1$; NIpassives: $F(6,80)=1.65, P>0.1)$. There was also no triple block order $\times$ group $\times$ target type interaction (idioms: $F<1$; Pidioms: $F(6,80)=1.82, P>0.1$; NI-actives: $F<1$; NI-passives: $F<1)$.

Performance accuracy was analysed along the same contrasts. Performance in HD and controls was similar in the analysis by subjects (controls: $99.34 \% \pm 2.62$ correct, HD: $96.87 \% \pm 10.02$ correct; $F 1(1,46)=3.74, P=0.06)$ but not in the analysis by items $(F 2(1,108)=54.47, P<0.001)$. There was a target type effect only in the analysis by items (word related $98.80 \% \pm 3.56$ correct, idiom-related $98.49 \% \pm 5.99$ correct, unrelated $97.03 \% \pm 10.73$ correct; $F 2$ (2, $108)=9.49, P<0.001)$ but not in the analysis by subjects $(F 1(2$, $92)=2.09, P>0.1)$. There was a prime type effect only in the analysis by items (idioms $98.68 \% \pm 4.30$ correct, P-idioms $98.89 \% \pm 3.94$ correct, NI-actives $97.64 \% \pm 8.93$ correct, NIpassives $97.22 \% \pm 10.27$ correct; $F 2(3,108)=5.16, P=0.002)$ but not in the analysis by subjects $(F 1(3,138)=2.49, P=0.06)$. There was no prime type $\times$ target type interaction $(F 1(6$, $276)=1.02, P>0.1 ; F 2(6,108)=1.23, P>0.1)$ nor a triple group $\times$ prime type $\times$ target type interaction $(F 1(6,276)=1.55$, $P>0.1 ; F 2(6,108)=2.10, P>0.1)$.

\subsection{Sub-set}

We used the two independent variables "group" (HD, controls) and "semantic restriction" (SR+, SR-). Fig. 2 summarises the results in controls and HD patients. RT were slower in HD patients $(869 \mathrm{~ms} \pm 154)$ than in controls $(559 \mathrm{~ms} \pm 101)$ $(F 1(1,46)=78.94, P<0.001 ; F 2(1,28)=226.26, P<0.001)$. In both controls and HD patients SR - yielded slower RT than SR+ sentences $(F 1(1,46)=56.80, P<0.001 ; F 2(1,28)=7.18$, $P=0.01)$ with no group $\times$ semantic restriction interaction $(F 1(1$, 46) $=2.70, P>0.1 ; F 2<1)$.

Analyses on performance accuracy were conducted similar to RT. Performance was lower in HD $(94.17 \% \pm 5.26$ correct $)$ than in controls $(98.75 \% \pm 2.63$ correct $)(F 1(1,46)=26.01$, $P<0.001 ; F 2(1,28)=8.47, P<0.01)$ but did not differ with SRand SR+ sentences $(F 1(1,46)=1.55, P>0.1 ; F 2<1)$. There was no group $\times$ semantic restriction interaction $(F 1(1,46)=2.56$, $P>0.1 ; F 2<1)$.

\section{Correlation analyses}

The experimental results of HD patients were correlated with the MDRS and different disease progression parameters of the UHDRS including executive function scores (Stroop test, word fluency, symbol digit code), motor scores and TFC (total functional capacity). Correlation analyses with syntactic rule application used the amount of priming with P-idioms 
Table 5

Correlation analyses in HD patients

\begin{tabular}{|c|c|c|c|c|c|c|c|c|c|}
\hline & \multicolumn{5}{|c|}{ Disease progression } & \multicolumn{4}{|l|}{ Sentence processing } \\
\hline & \multirow[t]{2}{*}{ TFC } & \multirow[t]{2}{*}{ UHDRS motor } & \multicolumn{3}{|c|}{ Executive functions } & \multicolumn{3}{|l|}{ Rule application } & \multirow{2}{*}{$\begin{array}{l}\text { Lexical processes } \\
\text { Idioms idiom-rel }\end{array}$} \\
\hline & & & Word fluency & Symbol digit test & Stroop W/C & $P$-idioms idiom-rel & $P$-idioms word-rel & NI-passives word-rel & \\
\hline \multicolumn{10}{|c|}{ MDRS } \\
\hline$R$ & $0.577 *$ & $0.532 *$ & $0.450^{*}$ & $0.638^{*}$ & $0.644^{*}$ & 0.029 & $0.555^{*}$ & 0.042 & 0.296 \\
\hline$P$ & 0.004 & 0.009 & 0.027 & 0.001 & 0.001 & 0.896 & 0.006 & 0.851 & 0.160 \\
\hline \multicolumn{10}{|c|}{ TFC } \\
\hline$R$ & & $0.420 *$ & $0.408 *$ & $0.636^{*}$ & $0.567 *$ & 0.240 & 0.201 & 0.303 & 0.353 \\
\hline$P$ & & 0.041 & 0.048 & 0.001 & 0.004 & 0.258 & 0.347 & 0.150 & 0.091 \\
\hline \multicolumn{10}{|c|}{ UHDRS motor } \\
\hline$R$ & & & $0.427 *$ & $0.708 *$ & $0.666^{*}$ & 0.085 & 0.105 & 0.140 & 0.363 \\
\hline$P$ & & & 0.037 & 0.000 & 0.000 & 0.693 & 0.825 & 0.515 & 0.081 \\
\hline \multicolumn{10}{|c|}{ Word fluency } \\
\hline$R$ & & & & $0.485^{*}$ & $0.652^{*}$ & 0.325 & 0.247 & 0.011 & 0.369 \\
\hline$P$ & & & & 0.016 & 0.001 & 0.121 & 0.244 & 0.958 & 0.076 \\
\hline \multicolumn{10}{|c|}{ Symbol digit code } \\
\hline$R$ & & & & & $0.740^{*}$ & 0.037 & $0.444 *$ & 0.110 & 0.353 \\
\hline$P$ & & & & & 0.000 & 0.862 & 0.030 & 0.610 & 0.090 \\
\hline \multicolumn{10}{|c|}{ Stroop W/C } \\
\hline$R$ & & & & & & 0.204 & 0.244 & 0.081 & 0.383 \\
\hline$P$ & & & & & & 0.340 & 0.251 & 0.708 & 0.065 \\
\hline
\end{tabular}

$R$, correlation coefficient; $P *$ is significant at the 0.05 level (two-tailed). TFC: total functional capacity; UHDRS motor: UHDRS motor scores; word fluency: word fluency (PRV) in 2 min; Stroop W/C: word-colour sub-part of the Stroop test; idiom-rel: idiom-related targets; word-rel: word-related targets. 
and NI-passives: (1) P-idioms ( $\mathrm{RT}_{\text {unrelated }}-\mathrm{RT}_{\text {idiom-related }}$ ), (2) P-idioms ( $\left.\mathrm{RT}_{\text {unrelated }}-\mathrm{RT}_{\text {word-related }}\right)$, (3) NI-passives $\left(\mathrm{RT}_{\text {unrelated }}-\mathrm{RT}_{\text {word-related }}\right)$. Correlation analyses with lexical abilities used the amount of priming reflecting access to idiom meanings: idioms ( $\left.\mathrm{RT}_{\text {unrelated }}-\mathrm{RT}_{\text {idiom-related }}\right)$.

Disease progression scores (TFC, UHDRS motor scores, executive function scores) and global intellectual capacities (MDRS) correlated with one another. Executive function scores did not correlate with either priming effects reflecting syntactic rule application (except for the symbol digit code and P-idioms $\left.\left(\mathrm{RT}_{\text {unrelated }}-\mathrm{RT}_{\text {word-related }}\right)\right)$ nor with priming effects reflecting lexical access to idiom meanings. Results are detailed in Table 5.

\section{Discussion}

We investigated the role of the striatum in sentence perception by testing the claim that the striatum specifically subserves syntactic rule use as opposed to lexical processes. Both aspects of sentence processing were assessed in a model of striatal disorders, namely early stages of HD. Using a priming paradigm we tested both the application of syntactic movement rules and lexical access to word meanings, to semantic restrictions and to idiom meanings.

Controls had lexical access to word meanings and to whole idiomatic sentences as shown by priming respectively for wordrelated targets with all sentence types and for idiom-related targets with idioms. In addition, they had access to semantic restrictions as reflected by increased reaction times with sentences containing semantic restriction violations. Finally, controls applied syntactic movement rules to passive structures as reflected by the disruption of priming for idiom-related targets with P-idioms and decreased priming for word-related targets with P-idioms and NI-passives. HD patients behaved like controls with respect to idioms and NI-actives. In contrast, they did not apply movement rules to passive sentence structures as reflected by persisting priming for idiom-related targets with $\mathrm{P}$ idioms and undecreased priming for word-related targets with P-idioms and NI-passives.

Taken together, our results indicate that the processing of phrasal structures relies on both lexical access to stored language representations and the application of syntactic rules. In $\mathrm{HD}$, lexical abilities are preserved whereas the application of syntactic movement rules is deficient. Indeed, HD patients conserve the ability to process idioms and simple active sentences but are impaired on the processing of passive structures. More specifically, our results suggest that HD patients process the non-canonical structure of passives along what could be called a linear "default rule", which permits the parsing of active sentences (first noun of the sentence $=$ agent role; last noun $=$ theme role). This kind of default rule applied whenever HD patients encountered passive structures independently from the sentence position within a given block and independently from the presentation order of the different sentence blocks. This finding is reminiscent of results provided in the domain of conjugation showing that HD patients apply a basic default rule to French non-verbs while they are impaired with non-verb stimuli that respond to less frequent sub-regularities (Teichmann et al., 2005).

Our results suggest that the striatum holds a role in sentence comprehension that pertains to the application of syntactic movement rules whereas it is not involved in lexical processing aspects. At first view, this supports the broad claim that the striatum is involved in the computation of language rules (Ullman, 2001) extending evidence from the word domain (Teichmann et al., 2005; Ullman et al., 1997) to the domain of phrasal structures. However, the striatum seems to subserve complex movement rules in syntax (see also Kemmerer, 1999; McNamara et al., 1996; Natsopoulos et al., 1993), whereas it was claimed to impact on more basic default rules in word morphology (Ullman et al., 1997). Thus, assuming that both complex and default rules represent genuine language rules in that they intervene whenever linguistic memory fails (Jackendoff, 2002; Pinker, 1999), the striatum appears to underpin distinct rule computations in distinct language domains. On the contrary, it may be argued that, independent from the language domain, the striatum impacts on both complex and default rules, yet at different degrees. Indeed, several studies have shown that striatal damage prominently impairs the computation of complex and infrequent rules, such as syntactic movement (Kemmerer, 1999; McNamara et al., 1996; Natsopoulos et al., 1993; Teichmann et al., 2005) and morphological sub-regularities (Teichmann et al., 2005), while more automated default rules are more mildly affected (Teichmann, Dupoux, Kouider, \& Bachoud-Lévi, 2006). Alternatively, it has been proposed that striatal damage mainly impacts on default rules, leading to excessive rule use in HD patients, who were shown to over-regularise during verb conjugation producing forms like dig-ed (from to dig) instead of dug (Ullman et al., 1997). In line with this, HD patients may basically have difficulties in inhibiting the over-active default rule, which in turn, might result in the diminished application of more infrequent rule operations. A similar view has been adopted by Longworth et al. (2005) who stated that the striatum is involved in general processes of inhibition that may account for language deficits with respect to morphology and syntax. Yet, even if the present data do not decisively demonstrate whether the striatum is involved in processes of default rule inhibition or whether it primarily impacts the application of complex language rules, some clarifying evidence is available from the domain of word morphology. Teichmann et al. (2006) assessing the perception of conjugated verb forms showed that HD patients were impaired in the use of both complex sub-rules and the default rule suggesting that the striatum comprises computational properties that are critical for rule application as such. Yet, sub-rules were more affected than the default rule indicating that there is a functional gradient within the striatum that might relate to the progression of neural degeneration following a dorsoventral/medio-lateral gradient (Vonsattel et al., 1985). Likewise, it might be that syntactic rule impairments in HD extend from syntactic movement to canonical word order processing as neural degeneration proceeds. Assessing the different syntactic rule abilities in different stages of HD should help to confirm the view of a genuine, but probably graduated, impairment on rule operations. 
In each case, our findings are at odds with the view stating that the striatum impacts the processing of lexical information. Friederici and Kotz (2003) and Longworth et al. (2005) proposed that the striatum is involved in late integrational processes that check whether lexicalised information is compatible with the syntactic frame. Such kinds of lexical checking processes were claimed to relate to controlled post-linguistic operations rather than to implicit language-specific processes. A similar proposal was formulated by Grossman et al. (2002) who claimed that the striatum impacts on sentence processing via its implication in general control processes that are tied to executive functions. However, our results challenge this lexical and nonlanguage specific view showing that HD patients had normal access to lexical information, such as word and idiom representations and semantic restrictions, by means of an implicit manipulation task that minimised executive-controlled response strategies. Yet, despite the short interval between the target onset and the critical word of the prime sentence, namely $200 \mathrm{~ms}$, one cannot exclude that the participants might have established explicit links between the primes and the targets. Such explicit processes could have modified the response strategy along the task and might have induced performance biases. To check for this, we ran post-hoc analyses showing that priming effects were independent from both the sentence position within a given block and the presentation order of the blocks. Moreover, we ran a series of correlation analyses showing that only one executive parameter among nine correlated with performance on syntactic rule application suggesting that rule impairment and executive damage evolve independently in HD.

In summary, our results show that the application of syntactic movement rules is deficient in HD whereas lexical processing at the phrasal level is not. Thus it is suggested that the striatum is involved in the former processes but not in the latter. Furthermore, the use of an implicit manipulation task and the absence of both prime order and block order effects as well as the near absence of correlations between executive and rule parameters suggest that the striatum subserves rule application via language-specific operations. Yet, we do not preclude the possibility that the striatum, which is known to encompass functional circuits of executive functioning (e.g., Middleton \& Strick, 2000), also controls lexical output at some post-linguistic level as implied by the data of Friederici et al. (1999) and Friederici, Kotz et al. (2003) and Kotz et al. (2003). This view is also coherent with findings showing that lexical operations such as word retrieval depend on controlled research strategies in the mental lexicon involving prefrontal and striatal structures (e.g., Rosen, Ojemann, Ollinger, \& Petersen, 2000). The same kinds of controlled processes might also account for the fact that striatal damaged patients have difficulties in explicit tasks assessing verbal fluency (e.g., Butters, Wolfe, Granholm, \& Martone, 1986) and object naming (e.g., Frank et al., 1996) and that they produce semantic/lexical paraphasias (Damasio et al., 1982). In an effort to conciliate both points of view, we propose that the striatum might hold a twofold role in language processing comprising controlled processes of lexical access via the link to executive areas and implicit rule computations via more language specific circuits. This proposal is compatible with recent findings from diffusion tensor imaging (Lehéricy et al., 2004) showing that the human striatum is connected to both the dorsal-lateral prefrontal cortex, known to subserve executive functions (e.g., Stuss \& Knight, 2002), and to portions of Broca's area, which is claimed to impact on combinatorial aspects of language processing (e.g., Hagoort, 2005). Likewise, it is compatible with data of implicit manipulation tasks showing that rule use in artificial grammar learning (Lieberman, Chang, Chiao, Bookheimer, \& Knowlton, 2004) and in word morphology (Teichmann et al., 2006) are tied to the striatum whereas lexical operations are not (Heindel, Salmon, Shults, Walicke, \& Butters, 1989; Salmon, Shimamura, Butters, \& Smith, 1988; Shimamura, Salmon, Squire, \& Butters, 1987). In particular, Lieberman et al. (2004) using fMRI showed that the striatum is activated when healthy adults abstract rules from letter strings even though they have no conscious insight into their inherent rule structure. Similarly, in morphology, Teichmann et al. (2006) assessed HD patients with a lexical decision task (word versus non-word decision), in which, unknown to the participants, the frequency of regular and irregular inflected verb forms was manipulated. HD patients applied rules merely to low frequency regulars while they accessed high frequency forms directly in the mental lexicon, suggesting that striatal disorders hamper implicit processes of rule application.

Altogether, this study provided novel arguments for the involvement of the striatum in language rule application through investigating the domain of sentence processing. In particular, we suggest that certain portions of the striatum are involved in language-specific computations that are related to rule application but not to lexical operations. Conversely, the striatum might control lexical operations via other functional circuits that are tied to executive functioning. Such a twofold role should be further verified by providing more detailed correlations between the different portions of the striatum and their respective involvement in the different language components.

On the other hand, our data showed that the role of the striatum in rule processing might be more restricted than initially claimed by the declarative-procedural model (Ullman, 2001). Indeed, its computational function seems to pertain to linguistic rules that govern syntactic movement whereas more basic rules defining the processing of simple active sentences are preserved. Further studies are necessary to evaluate this more fine-grained distinction and to track the specific kind of language rules that are subserved by striatal structures.

\section{Acknowledgments}

This work was conducted with the help of an Avenir grant (2001) allocated to Bachoud-Lévi by the INSERM and an Assistant Hospitalier de Recherche (AHR AP-HP/INSERM) et Fondation pour la Recherche Médicale grants to Marc Teichmann. We thank Marie-Françoise Boissé for assessing MDRS scores and cognitive scales of the UHDRS and Guillaume Dolbeau and Amandine Rialland for transmitting the data. Furthermore we wish to thank the Neurology Department of the Henri Mondor Hospital and the CIC for providing access to the patients. We are grateful to the Réseau Huntington de Langue 
Française who supports this work. We wish also to thank Helen Bates and Chantal Vachon for the corrections of the manuscript.

\section{References}

Butters, N., Wolfe, J., Granholm, E., \& Martone, M. (1986). An assessment of verbal recall, recognition and fluency abilities in patients with Huntington's disease. Cortex, 22, 11-32.

Cacciari, C., \& Tabossi, P. (1988). The comprehension of idioms. Journal of Memory and Language, 27, 668-683.

Cardebat, D., Doyon, B., Puel, M., Goulet, P., \& Joanette, Y. (1990). Formal and semantic lexical evocation in normal subjects. Performance and dynamics of production as a function of sex, age and educational level. Acta Neurologica Belgica, 90, 207-217.

Chomsky, N. (1965). Aspects of the theory of syntax. Cambridge: MIT Press.

Chomsky, N. (1977). On Wh movement. In P. Culicover, T. Wasow, \& A. Akmajian (Eds.), Formal syntax. New York: Academic Press.

Chomsky, N. (1980). Rules and representations. New York: Columbia University Press.

Chomsky, N. (1986). Barriers. Cambridge, MA: MIT Press.

Copland, D. (2003). The basal ganglia and semantic engagement: Potential insights from semantic priming in individuals with subcortical vascular lesions, Parkinson's disease, and cortical lesions. Journal of the International Neuropsychological Society, 9, 1041-1052.

Damasio, A. R., Damasio, H., Rizzo, M., Varney, N., \& Gersh, F. (1982). Aphasia with non-hemorrhagic lesions in the basal ganglia and internal capsule. Archives of Neurology, 39, 15-24.

Frank, E. M., McDade, H. L., \& Scott, W. K. (1996). Naming in dementia secondary to Parkinson's, Huntington's, and Alzheimer's diseases. Journal of Communication Disorders, 29, 183-197.

Friederici, A., \& Kotz, S. A. (2003). The brain basis of syntactic processes: Functional imaging and lesion studies. Neuroimage, 20(Suppl 1), 8-17.

Friederici, A. D., Kotz, S. A., Werheid, K., Hein, G., \& von Cramon, D. Y. (2003). Syntactic comprehension in Parkinson's disease: Investigating early automatic and late integrational processes using event-related brain potentials. Neuropsychology, 17, 133-142.

Friederici, A., Ruschemeyer, S. A., Hahne, A., \& Fiebach, C. J. (2003). The role of left inferior frontal and superior temporal cortex in sentence comprehension: Localising syntactic and semantic processes. Cerebral Cortex, 13(2), 70-77.

Friederici, A. D., von Cramon, D. Y., \& Kotz, S. A. (1999). Language related brain potentials in patients with cortical and subcortical left hemisphere lesions. Brain, 122, 1033-1047.

Golden, C. J. (1978). Stroop color and word test. Chicago: Stoelting.

Grossman, M., Kalmanson, J., Bernhardt, N., Morris, J., Stern, M. B., \& Hurtig, H. I. (2000). Cognitive resource limitations during sentence comprehension in Parkinson's disease. Brain and Language, 73, 1-16.

Grossman, M., Zurif, E., Lee, C., Prather, P., Kalmanson, J., Stern, M. B., et al. (2002). Information processing speed and sentence comprehension in Parkinson's disease. Neuropsychology, 16, 174-181.

Heindel, W. C., Salmon, D. P., Shults, C. W., Walicke, P. A., \& Butters, N. (1989). Neuropsychological evidence for multiple implicit memory systems: A comparison of Alzheimer's, Huntington's, and Parkinson's disease patients. The Journal of Neuroscience: The Official Journal of the Society for Neuroscience, 9(2), 582-587.

Hagoort, P. (2005). On Broca, brain, and binding: A new framework. Trends in Cognitive sciences, 9(9), 416-423.

Huntington Study Group. (1996). Unified Huntington's disease rating scale: Reliability and consistency. Movement Disorders: Official Journal of the Movement Disorder Society, 11, 136-142.

Illes, J. (1989). Neurolinguistic features of spontaneous language production dissociate three forms of neurodegenerative disease: Alzheimer's, Huntington's, and Parkinson's. Brain and Language, 37, 628-642.

Jackendoff, R. (2002). Foundations of language: Brain, meaning, grammar, evolution. New York: Oxford University Press.

Kemmerer, D. (1999). Impaired comprehension of raising-to-subject constructions in Parkinson's disease. Brain and Language, 66, 311-328.
Kotz, S. A., Frisch, S., von Cramon, D. Y., \& Friederici, A. D. (2003). Syntactic language processing: ERP lesion data on the role of the basal ganglia. Journal of the International Neuropsychological Society, 9, 1053-1060.

Kuhl, D. E., Phelps, M.E., Markham, C. H., Metter, E. J., Riege, W. H., \& Winter, J. (1982). Cerebral metabolism and atrophy in Huntington's disease determined by $18 \mathrm{FDG}$ and computed tomographic scan. Annals of Neurology, $12,425-434$.

Lehéricy, S., Ducros, M., Van de Moortele, P. F., Francois, C., Thivard, L., Poupon, C., et al. (2004). Diffusion tensor fiber tracking shows distinct corticostriatal circuits in humans. Annals of Neurology, 55, 522-529.

Lieberman, M. D., Chang, G. Y., Chiao, J., Bookheimer, S. Y., \& Knowlton, B. J. (2004). An event-related fMRI study of artificial grammar learning in a balanced chunk strength design. Journal of Cognitive Neuroscience, 16(3), $427-438$.

Longworth, C. E., Keenan, S. E., Barker, R. A., Marslen-Wilson, W. D., \& Tyler, L. K. (2005). The basal ganglia and rule-governed language use: Evidence from vascular and degenerative conditions. Brain, 128, 584-596.

Macdonald, M. C., Pearlmutter, N. J., \& Seidenberg, M. S. (1994). Lexical nature of syntactic ambiguity resolution. Psychological Review, 101, 676-703.

Mattis, S. (1976). Mental status examination for organic mental syndrome in elderly patients. In L. Bellak \& T. B. Karasu (Eds.), Geriatric psychiatry (pp. 77-121). New York: Grune \& Straton.

Mazziotta, J. C., Phelps, M. E., Pahl, J. J., Huang, S. C., Baxter, L. R., Riege, W. H., et al. (1987). Reduced cerebral glucose metabolism in asymptomatic subjects at risk for Huntington's disease. The New England Journal of Medicine, $316,357-362$.

McNamara, P., Krueger, M., O’Quin, K., Clark, J., \& Durso, R. (1996). Grammaticality judgments and sentence comprehension in Parkinson's disease: A comparison with Broca's aphasia. The International Journal of Neuroscience, 86, 151-166.

Middleton, F. A., \& Strick, P. L. (2000). Basal ganglia and cerebellar loops: Motor and cognitive circuits. Brain Research. Brain Research Reviews, 31 , 236-250.

Moro, A., Tettamanti, M., Perani, D., Donati, C., Cappa, S. F., \& Fazio, F. (2001). Syntax and the brain: Disentangling grammar by selective anomalies. Neuroimage, 13, 110-118.

Natsopoulos, D., Grouios, G., Bostantzopoulou, S., Mentenopoulos, G., Katsarou, Z., \& Logothetis, J. (1993). Algorithmic and heuristic strategies in comprehension of complement clauses by patients with Parkinson's disease. Neuropsychologia, 31, 951-964.

New, B., Pallier, C., Brysbaert, M., \& Ferrand, L. (2004). Lexique 2: A new French lexical database. Behavior Research Methods, Instruments \& Computers, 36(3), 516-524.

Peschanski, M., Cesaro, P., \& Hantraye, P. (1995). Rationale for intrastriatal grafting of striatal neuroblasts in patients with Huntington's disease. Neuroscience, 68, 273-285.

Pinker, S. (1999). Words and rules: The ingredients of language. New York: Basic Books.

Rosen, H. J., Ojemann, J. G., Ollinger, J. M., \& Petersen, S. E. (2000). Comparison of brain activation during word retrieval done silently and aloud using FMRI. Brain and Cognition, 42, 201-217.

Salmon, D. P., Shimamura, A. P., Butters, N., \& Smith, S. (1988). Lexical and semantic priming deficits in patients with Alzheimer's disease. Journal of Clinical and Experimental Neuropsychology: Official Journal of the International Neuropsychological Society, 10, 477-494.

Schriefers, H., Friederici, A. D., \& Rose, U. (1998). Context effects in visual word recognition: Lexical relatedness and syntactic context. Memory \& Cognition, 26, 1292-1303.

Shimamura, A. P., Salmon, D. P., Squire, L. R., \& Butters, N. (1987). Memory dysfunction and word priming in dementia and amnesia. Behavioral Neuroscience, 101(3), 347-351.

Shoulson, I. (1981). Huntington disease: Functional capacities in patients treated with neuroleptic and antidepressant drugs. Neurology, 31, 1333-1335.

Stuss, D., \& Knight, R. T. (2002). Principles of frontal lobe function. New York: Oxford University Press.

Swinney, D. A., \& Cutler, A. (1979). The access and processing of idiomatic expressions. Journal of Verbal Learning and Verbal Behavior, 18, 523534. 
Teichmann, M., Dupoux, E., Kouider, S., Brugières, P., Boissé, M. F., Baudic, S., et al. (2005). The role of the striatum in rule application: The model of Huntington's disease at early stage. Brain, 128, 1155-1167.

Teichmann, M., Dupoux, E., Kouider, S., \& Bachoud-Lévi, A. C. (2006). The role of the striatum in processing language rules: Evidence from word perception in Huntington's disease. Journal of Cognitive Neuroscience, 18(9), $1-15$.

Tettamanti, M., Moro, A., Messa, C., Moresco, R. M., Rizzo, G., Carpinelli, A., et al. (2005). Basal ganglia and language: Phonology modulates dopaminergic release. Neuroreport, 16(4), 397-401.

Titone, D. A., \& Connine, C. M. (1994). The comprehension of idiomatic expressions: Effects of predictability and literality. Journal of Experimental Psychology. Learning, Memory, and Cognition, 20, 1126-1138.

Tyler, L. K. (1985). Real-time comprehension processes in agrammatism: A case study. Brain and Language, 26, 259-275.

Ullman, M. T. (2001). A neurocognitive perspective on language: The declarative/procedural model. Nature Reviews. Neuroscience, 2(10), 717-726.
Ullman, M. T., Corkin, S., Coppola, M., Hickok, G., Growdon, J. H., Koroshetz, W. J., et al. (1997). A neural dissociation within language: Evidence that the mental dictionary is part of declarative memory, and that grammatical rules are processed by the procedural system. Journal of Cognitive Neuroscience, 9(2), 266-276.

Vannest, J., Polk, T. A., \& Lewis, R. L. (2005). Dual-route processing of complex words: New fMRI evidence from derivational suffixation. Cognitive, Affective \& Behavioral Neuroscience, 5(1), 67-76.

Vonsattel, J. P., Myers, R. H., Stevens, T. J., Ferrante, R. J., Bird, E. D., \& Richardson, E. P., Jr. (1985). Neuropathological classification of Huntington's disease. Journal of Neuropathology and Experimental Neurology, 44, 559-577.

Wallesch, C. W., \& Papagno, C. (1988). Subcortical aphasia. In F. C. Rose, R. Whurr, \& M. Wyke (Eds.), Aphasia (pp. 256-287). London: Whurr Publishers.

Wechsler, D. (1981). Wechsler adult intelligence scale-revised manual. New York: Psychological Corporation. 